\title{
Dynamic double contrast MRI of the pelvic floor in patients with disorders of defecation
}

P.J. Dempsey, J.W. Power, C. McEntee, J. Bisset, A.E. Brannigan, J.J. Mulsow,

T. Geoghegan, M.M. McNicholas - Mater Misericordiae University Hospital Dublin

\section{Anatomy}

The pelvic floor is a musculofascial structure inserted into the pelvic bones which serves several functions.

- Closes and controls pelvic outlet

- Provides static support against gravity and intraabdominal pressure

- Controls continence and evacuation

\section{Pelvic diaphragm \\ - Levator ani \\ - Puborectalis \\ - lliococcygeus \\ - Pubococcygeus}

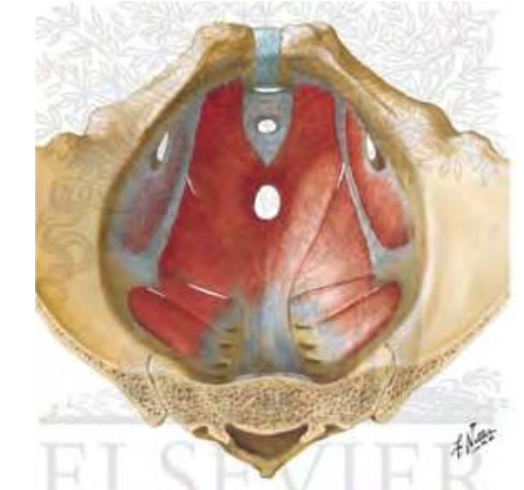

Definition of prolapse

Abnormal descent of a pelvic organ through the hiatus beneath it

- Cystoceole

- Peritinoceole

- Enterocoele

- Uterine prolapse

- Vaginal prolapse

- Sigmoidocoele

- Rectal prolapse

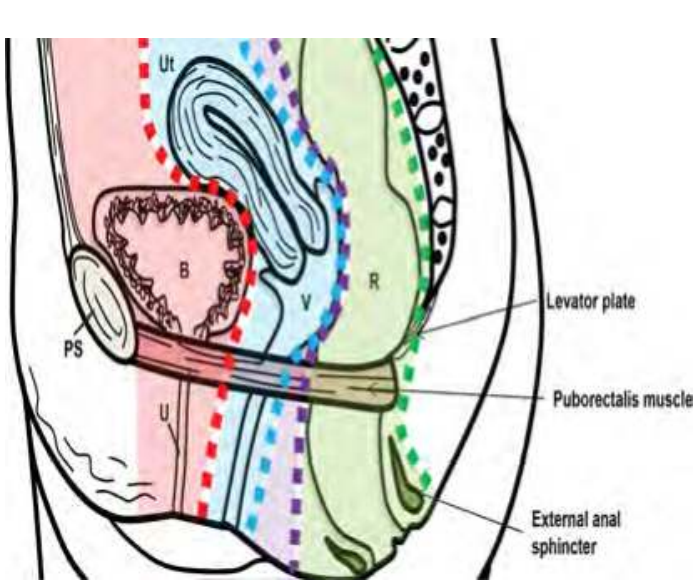

MRI technique

$50 \mathrm{cc}$ ultrasound gel self administered into rectum and vagina. Entire study 15 mins.

Static images

- Localiser

- T1 whole pelvis axial

- T2 TSE Cor Small FOV

- T2 TSE Axial Small FOV

Dynamic series in sag plane

- T2 Haste resting

- T2 Haste clenching

- Cine loop: (Resting/clenching/ bearing down)

- T2 Haste bearing down
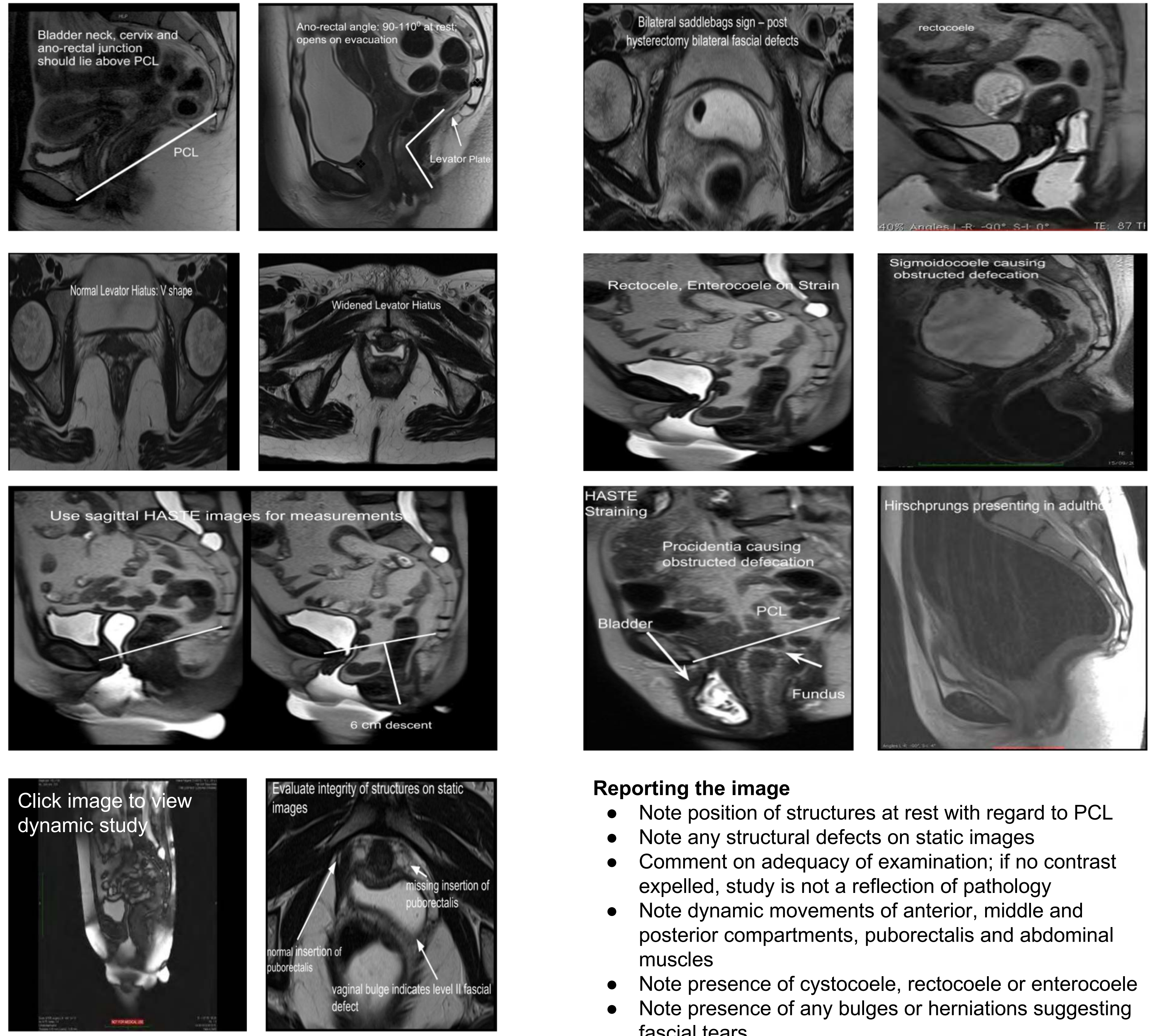
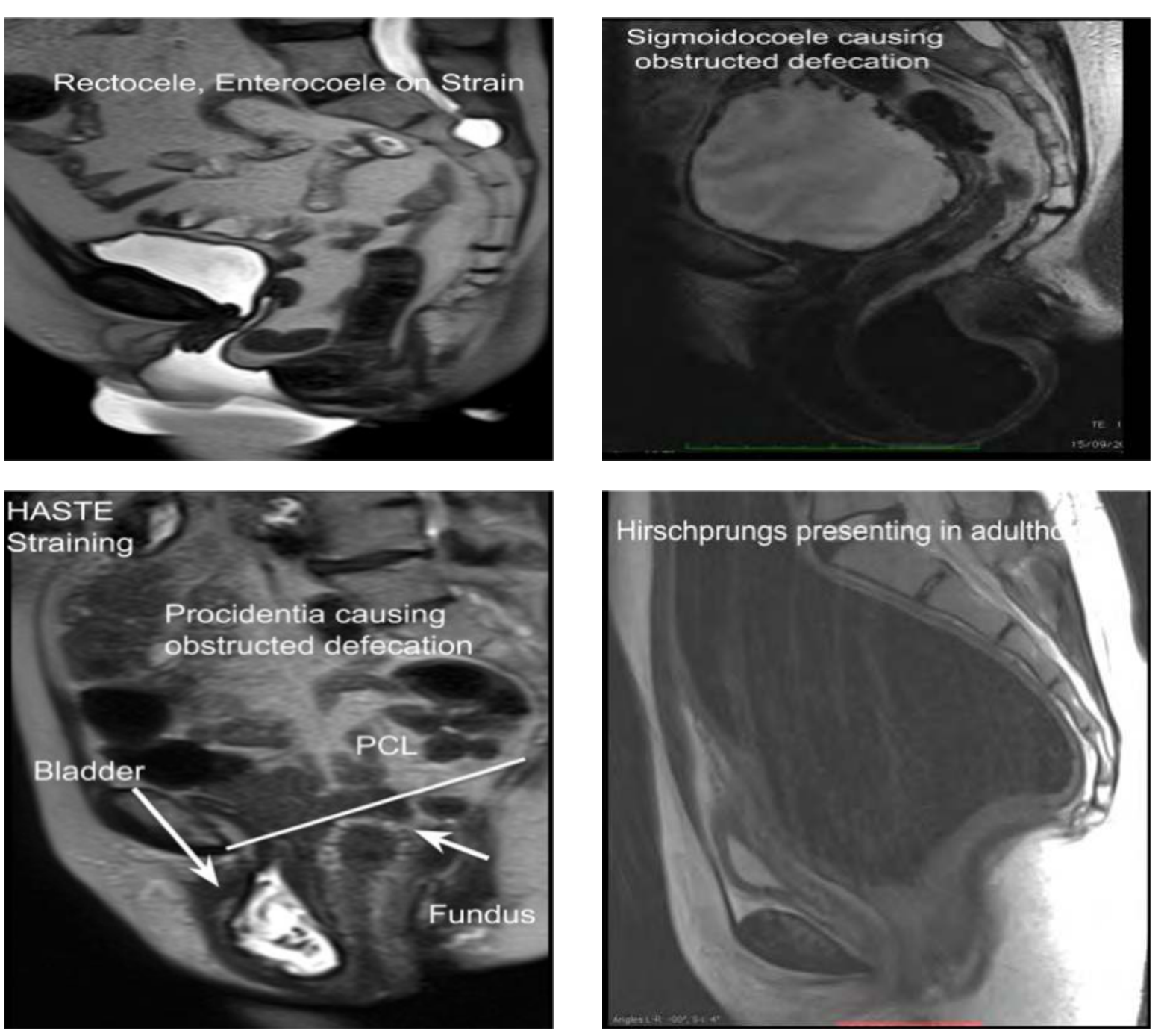

Reporting the image

- Note any structural defects on static images

- Comment on adequacy of examination; if no contrast expelled, study is not a reflection of pathology

- Note dynamic movements of anterior, middle and posterior compartments, puborectalis and abdominal muscles

- Note presence of cystocoele, rectocoele or enterocoele

- Note presence of any bulges or herniations suggesting fascial tears

- Note urethral motility
- Note position of structures at rest with regard to PCL 